\title{
Lessons from the heart: urgent evaluation and treatment of cerebral ischemia
}

\author{
Argye E Hillis
}

Consider two common scenarios. In the first, Mrs Smith experiences sudden weakness of the right arm and face and mild difficulty speaking. She calls her primary care physician, but when her call is returned 7 hours later, her symptoms have resolved. The physician diagnoses a transient ischemic attack (TIA), recommends a daily aspirin for stroke prevention, and refers Mrs Smith to a neurologist. Six weeks later, the neurologist orders a carotid ultrasound and echocardiogram. The ultrasound, completed 1 week later, shows $80-89 \%$ carotid stenosis, which is confirmed the following week by magnetic resonance angiography. A vascular surgeon recommends carotid endarterectomy (CEA), which is finally performed 12 weeks after the TIA.

In the second scenario, Mrs Brown experiences sudden-onset crushing chest pain and shortness of breath. She calls for an ambulance and is admitted to hospital, where tests confirm cardiac ischemia. She undergoes a cardiac catheterization the following day, which identifies $90 \%$ stenosis of the left anterior descending artery. Mrs Brown undergoes urgent cardiac bypass surgery the next day, 48 hours after the onset of chest pain.

In this issue, Schneck and Biller, commenting on a recent study by Fairfield and colleagues, argue that this discrepancy in treatment timing is unjustifiable. The data in their study indicate that Mrs Smith was lucky; she had a $32 \%$ risk of stroke and a $16 \%$ risk of disabling or fatal stroke in the interim between her symptom onset and CEA. The investigators also found that in Oxfordshire, UK, the mean time from TIA or stroke to carotid imaging was 33 days, and from the event to CEA was 100 days.

Previous studies have shown that CEA substantially reduces risk of recurrent stroke if completed within 2 weeks of the index event, but only minimally reduces risk if carried out 12 weeks after the event. In previous trials and audits of CEA in Europe and North America,
Ischemic

stroke should

be considered

an evolving

emergency,

bringing the

opportunity to

limit damage

and to restore

function

AE Hillis is an associate professor of neurology at Johns Hopkins University School of Medicine, Baltimore, MD, USA, and an Advisory

Board member of

Nature Clinical

Practice Neurology.

Competing interests

The author declared she has no competing interests.

www.nature.com/clinicalpractice doi:10.1038/ncpneuro0132 however, median delays from event to surgery were never within 2 weeks, and often exceeded 2 months.

What would be the impact of earlier treatment? Fairfield et al. estimate that surgery in 49 patients in Oxfordshire prevented five strokes, but, had CEA been performed within 2 weeks from the index event, 14 strokes could have been prevented. Extrapolating from these data, the 131,000 endarterectomies performed in 1999 in the US might have prevented as many as 13,357 strokes in the subsequent 5 years; however, had they all been performed within 2 weeks of presenting ischemic symptoms, as many as 37,428 strokes could have been prevented.

There is increasing awareness that stroke outcome can be improved by intravenous thrombolysis within 3 hours of onset, but it is not widely appreciated that emergency treatment of stroke after 3 hours can also have marked benefit. Urgent treatment of carotid stenosis with CEA or carotid stenting not only prevents stroke, but in some cases can also improve cognitive impairment, or even reverse the severe aphasia and hemiplegia caused by hypoperfusion. In patients with stroke caused by intracranial large-vessel stenosis, other interventions to restore blood flow, such as intra-arterial thrombolysis or induced temporary blood pressure elevation, can improve function and perhaps prevent extension of the infarct. Many disabling cardioembolic strokes might be prevented by rapid identification and treatment of arrhythmias or other cardioembolic sources of TIA or minor stroke. The effectiveness of all these interventions is greatest early after symptom onset.

Schneck and Biller urge that symptomatic carotid stenosis and TIA should be managed as 'unstable angina of the brain'. Furthermore, ischemic stroke should be considered an evolving emergency, bringing the opportunity to limit damage and to restore function. The brain needs the treatment the heart receives. 